Copyright (C) 2005 IEEE. Reprinted from

IEEE Transactions on Antennas and Propagation, 2005; 53 (10):3174-

3179

This material is posted here with permission of the IEEE. Such permission of the IEEE does not in any way imply IEEE endorsement of any of the University of Adelaide's products or services. Internal or personal use of this material is permitted. However, permission to reprint/republish this material for advertising or promotional purposes or for creating new collective works for resale or redistribution must be obtained from the IEEE by writing to pubs-permissions@ieee.org.

By choosing to view this document, you agree to all provisions of the copyright laws protecting it. 


\title{
A Kirchhoff Integral Approach to Estimating Propagation in an Environment With Nonhomogeneous Atmosphere and Complex Boundaries
}

\author{
Chris J. Coleman, Member, IEEE
}

\begin{abstract}
For terrestrial radio wave propagation, it has been previously demonstrated that second order effects, such as diffraction, can be estimated through a combination of geometric optics and Kirchhoff style integral relations. In the present paper, it is shown that the approach can also yield accurate estimates when refractive effects, such as atmospheric ducting, are present.
\end{abstract}

Index Terms-Atmospheric propagation, diffraction, Kirchhoff integral, ducting, reciprocity, refraction.

\section{INTRODUCTION}

$\mathbf{T}$ HE performance of radar and communications systems, at Gigahertz frequencies, can be severely affected by the environment in which they operate. Not only can ground topography greatly influence radio wave propagation through diffraction, but subtle variations in the atmosphere can have a severe impact through refraction. Because of this, radio system planners require effective tools that allow them to assess the impact of propagation upon system performance. This report describes some new techniques that can be used to assess propagation for which both diffractive and refractive effects are important. Whilst much of the propagation is adequately described by geometric optics, there are regions, particularly at low altitudes, where such a procedure is inadequate. At low altitudes, direct propagation is limited by the curvature of the Earth and propagation mechanisms such as diffraction, ducting and ground reflections can dominate. Such mechanisms require a more comprehensive approach to Maxwell's equations than is afforded by geometric optics and suitable approaches are usually much more computationally expensive. The parabolic equation (PE) technique is one such approach, but requires the solution of a partial differential equation over a fairly refined grid. Significant savings, however, can be achieved by use of split step fast Fourier transform techniques. Additional savings can be made if the PE method is restricted to lower altitudes and then blended with geometric optics techniques [1]-[3] at greater heights. Such an approach is the basis of many current propagation models for microwave systems and [1] provides a general overview of PE techniques. Other increasingly popular approaches are provided by the finite-difference time-domain (FDTD) technique (see [4]-[6] for some examples of this approach) and the transmission line matrix (TLM) technique [7]. Reference [8] provides a general overview of most of the available approaches to microwave propagation.

The present paper explores an alternative procedure that is based upon a combination of geometric optics (GO) solutions and Kirchhoff style integral relations. These relations are derived from the reciprocity theorem, a theorem that allows one to probe an electromagnetic field with another quite unrelated field. In essence, the current work can be regarded as an extension of the ideas of physical optics [9]. An important property of the approach is that it allows one to generate second order estimates of the electromagnetic field from first order estimates (GO estimates for example). Using a simple form of this approach, Monteath [10] has shown that the effects of ground wave propagation, and diffraction, can be derived from GO solutions. The approach has been extended to more complex geometries in [11] and to propagation through inhomogeneous media in [12]. In the current paper, the emphasis is on phenomena that affect $\mathrm{GHz}$ frequencies. The paper describes how the basic approach can be extended to provide a means of estimating propagation in an environment that generates atmospheric ducting, as well as diffraction over obstacles and reflections from complex terrain. For weakly varying refractive index (such as in the lower atmosphere), the approach does not require the solution of ray tracing equations and hence results in an efficient propagation algorithm. The major advantage of the current approach is that it uses integral propagators to advance the solution and so the nature of the discretization can vary along the propagation path, taking advantage of local simplifications. Furthermore, the steplengths in the approximate solution can be very large and still retain good accuracy. These are major advantages for the current approach since alternatives such as the PDE, TLM or FDTD methods require fixed solution grids and small steplengths.

\section{BASIC APPROACH}

The author is with the Electrical and Electronic Engineering Department, The University of Adelaide, Adelaide 5005, South Australia (e-mail: ccoleman@eleceng.adelaide.edu.au).

Digital Object Identifier 10.1109/TAP.2005.856359 magnetic field $(\underline{E}, \underline{H})$ with sources outside Consider electromagnetic field $(\underline{E}, \underline{H})$ with sources outside
closed surface $S$ and another dipole field $\left(\underline{E}_{0}, \underline{H}_{0}\right)$ caused by 
a current element $\underline{J}_{0}$ at position $\underline{r}_{0}$. If $\underline{r}_{0}$ is contained within surface $S$ then

$$
\underline{E}\left(\underline{r}_{0}\right) \cdot \underline{J}_{0}=\int_{S}\left(\underline{E} \times \underline{H}_{0}-\underline{E}_{0} \times \underline{H}\right) \cdot d \underline{S} .
$$

Furthermore, if $\left(\underline{E}_{0}, \underline{H}_{0}\right)$ is now a dipole field generated by a magnetic current element $\underline{M}_{0}$ at position $\underline{r}_{0}$, then

$$
-\underline{H}\left(\underline{r}_{0}\right) \cdot \underline{M}_{0}=\int_{S}\left(\underline{E} \times \underline{H}_{0}-\underline{E}_{0} \times \underline{H}\right) \cdot d \underline{S} .
$$

These equations are a variant of the normal reciprocity result [10] and provide an integral formulation of the electromagnetic field equations. Furthermore, although the material properties of the medium do not appear explicitly, the equations will still apply to non homogeneous media. It is only required that the dipole fields satisfy the appropriate Maxwell equations for the non homogeneous medium. Now consider the sources of field $(\underline{E}, \underline{H})$ to be bounded, then the above equations will still apply if $S$ is an open surface that extends to infinity and separates the sources from the point $\underline{r}_{0}$ (the open surface can be closed by an additional surface at infinity on which the integrand disappears). If the fields are known on surface $S$, the above equations can be used to find the fields at point $\underline{r}_{0}$. As a consequence, on assuming only forward propagation to be significant, (1) and (2) can be used to advance an electro-magnetic field onwards from a surface $S$ on which it is known.

The only problem with the above approach is the absence of suitable dipole fields for media with other than very simple structure. In the case of homogeneous media, it has been shown that GO dipole fields can provide an effective development of the field [11]. Furthermore, the effects of the ground and structures can be taken into account by means of reflection coefficients. The GO solutions, however, will have a limited range of applicability and so (1) and (2) can only be used to develop the solution to within this range. To overcome this problem, we could use (1) and (2) to advance the fields to a new surface $S$ that is within the region of validity and then repeat the process at the new surface and so on until sufficient coverage has been achieved. Consequently, we can advance the fields on a series of surfaces $S_{1}$ to $S_{N}$ through the propagation region. Fig. 1 shows a typical problem that can be analyzed by such an approach. It consists of propagation that must travel from a transmitter located over water, surmount a building and then a hill. It is clear that there will be significant diffraction at the edges of the building, and the brow of the hill, and so we must place intermediate surfaces at these locations. It is advisable to place intermediate surfaces at all points for which there could be a break down in the GO solution. In particular, features such as corners, and changes in material properties, will require intermediate surfaces. Essentially, although the geometric optics solution breaks down in the immediate vicinity of these features, it is valid over the majority of each intermediate surface and hence is sufficient for the evaluation of the surface integrals. For near grazing incidence, the direct and reflected rays will nearly cancel and suitably positioned intermediate surfaces are

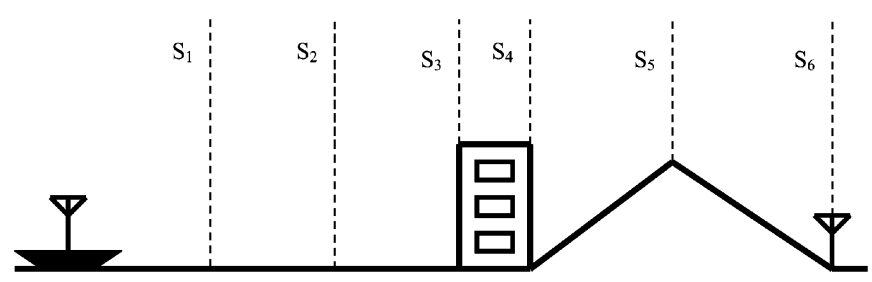

Fig. 1. Typical propagation scenario with suitable intermediate surfaces.

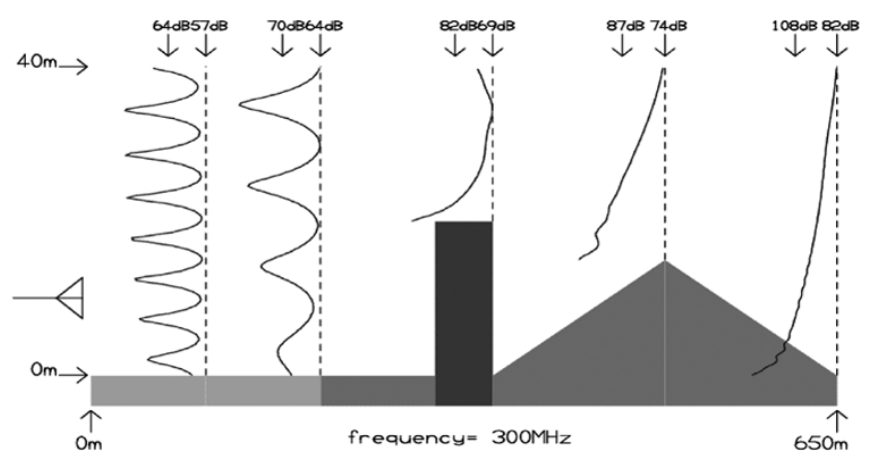

Fig. 2. Transmission losses experienced by signals in the scenario of Fig. 1.

required to pick up any ground wave effects. In essence, the repeated application of (1) and (2) is a generalization of the procedure used by Monteath to analyze simple ground wave propagation and diffraction by a screen.

Fig. 2 shows results from simulations of the basic transmission loss (transmitted power divided by the received power when the antennas are isotropic) experienced by the transmitted signal for the scenario of Fig. 1. (Note that the water has $\sigma=5 \mathrm{~S} / \mathrm{m}$ and $\varepsilon_{r}=80$, the building has $\sigma=0.001 \mathrm{~S} / \mathrm{m}$ and $\varepsilon_{r}=10$ and the ground has $\sigma=0.01 \mathrm{~S} / \mathrm{m}$ and $\varepsilon_{r}=10$ ). A particular loss is represented by its deviation from the vertical line that represents the appropriate intermediate surface. It will be noted that the approach predicts that power will be diffracted into the shadow regions behind both the building and the hill.

For many propagation problems, the propagation direction is roughly horizontal and, in this case, the integral equations can be approximated by

$$
\underline{E}\left(\underline{r}_{0}\right) \cdot \underline{J}_{0}=-2 \int_{S} \frac{\underline{E} \cdot \underline{E}_{0}}{\eta} d S
$$

where the electric field of the current element can be approximated by

$$
\underline{E}_{0} \approx \frac{j \omega \mu}{4 \pi} \underline{J}_{0} \frac{\exp (-j \beta \phi)}{s}
$$

for situations where the propagation does not involve ground reflections ( $\beta$ is the free-space wave-number, $\phi$ is the phase distance between the source and test point $\underline{r}$ and $s$ is the spreading distance). If ground reflections be present

$$
\begin{aligned}
\underline{E}_{0}(\underline{r}) & =\underline{E}_{0}^{d}(\underline{r})+\underline{E}_{0}^{r}(\underline{r}) \\
& \approx \frac{j \omega \mu}{4 \pi} \underline{J}_{0}\left(\frac{\exp \left(-j \beta \phi_{d}\right)}{s_{d}}+R \frac{\exp \left(-j \beta \phi_{r}\right)}{s_{r}}\right)
\end{aligned}
$$


where the subscripts $d$ and $r$ refer to the distances measured along the direct and reflected ray paths respectively (superscripts are used to identify the direct and reflected parts of the fields). Quantity $R$ is the standard plane wave reflection coefficient that is appropriate to the polarization of the field source.

\section{EVALUATION OF THE INTEGRALS}

In the case of propagation that only involves diffraction over simple objects (screens for example), the integrals of (1)-(3) can often be approximated by those of the Fresnel variety. In general, however, the integrals are far more complex and must be evaluated numerically. Unfortunately, such integrals are slow to converge and need to be taken to very high altitudes before they are sufficiently accurate (400 $\mathrm{m}$ in the simulation of Section II). Before the integrals start to converge, however, the integrand has usually settled into a form that can be evaluated by Fresnel related integrals. In the current study, the approach adopted for (1)-(3) is to evaluate the integral to this point and then to complete it by means of a suitable analytic result. At the point where the numerical procedure ends, both the phase and magnitude of the integrand are expanded as far as quadratic terms in the height $Y$ above this point

$$
\begin{aligned}
\frac{\underline{E} \cdot \underline{E}_{0}}{\eta} \approx\left(I_{0}+I_{1} Y\right. & \left.+I_{2} Y^{2}\right) \\
& \times \exp \left(-j \beta\left(\phi_{0}+\phi_{1} Y+\phi_{2} Y^{2}\right)\right)
\end{aligned}
$$

(coefficients of the amplitude and phase Taylor series are evaluated from suitable numerical derivatives of the integrand). Although the expansion of the amplitude has only a limited range of validity, the increasing frequency of oscillation for the exponential term will ensure that the amplitude has negligible effect upon the integral outside this range. Expansions of the form of (6) were introduced by Monteath in his consideration of ground waves [10]. The integral of the expansion is evaluated by means of the analytic results

$$
\begin{aligned}
M_{0}(a, b, c) & =\int_{0}^{\infty} \exp \left(-j\left(a Y^{2}+2 b Y+c\right)\right) d Y \\
& =\frac{1}{\sqrt{a}} \exp \left(j \frac{b^{2}-a c}{a}\right) \int_{b \sqrt{a}}^{\infty} \exp \left(-j V^{2}\right) d V \\
M_{1}(a, b, c) & =\int_{0}^{\infty} Y \exp \left(-j\left(a Y^{2}+2 b Y+c\right)\right) d Y \\
& =-\frac{b}{a} M_{0}(a, b, c)-\frac{j}{2 a^{2}} \exp (-j c)
\end{aligned}
$$

and

$$
\begin{aligned}
M_{2}(a, b, c) & =\int_{0}^{\infty} Y^{2} \exp \left(-j\left(a Y^{2}+2 b Y+c\right)\right) d Y \\
& =\left(\frac{b^{2}}{a^{2}}-\frac{j}{2 a}\right) M_{0}(a, b, c)+\frac{b j}{2 a^{2}} \exp (-j c) .
\end{aligned}
$$

All that is required for to complete the evaluation of the above expressions is the Fresnel integrals $C$ and $S$ since

$$
\int_{x}^{\infty} \exp \left(-j V^{2}\right) d V=\sqrt{\frac{\pi}{2}}(C(x)-j S(x)) .
$$

There exist some excellent rational approximations to $C$ and $S$ which can be found in the book by Abramowitz and Stegun [13]. In general, a similar approach can be adopted for integration in the lateral direction. The current work, however, assumes that the topography and atmospheric variations are slow enough for an asymptotic evaluation of lateral integrals. At an intermediate surface, its is assumed that the fields can be approximated as $\underline{E}=\underline{\hat{E}} \exp \left(-j \beta\left(x^{2} / 2 z\right)\right)$ where $z$ is the horizontal distance from the source, $x$ is the lateral distance from the direct path and $\underline{E}$ is the electric field for points above this path. Using such approximations in the integral equations, and integrating first with respect to the variable $x$, it can be shown that the total surface integral is obtained by multiplying the vertical integral on the direct propagation path by the factor $\sqrt{\lambda z_{+} z_{-} / j\left(z_{+}+z_{-}\right)}$ where $z_{+}$and $z_{-}$are the horizontal distances from the integration surface to the field source and observation points respectively.

For problems involving ground reflections, a further refinement was found to increase accuracy. Both $\underline{E}$ and $\underline{E}_{0}$ are separated into their direct and reflected components and the integrals that involve $\underline{E}^{d} \cdot \underline{E}_{0}^{d}, \underline{E}^{d} \cdot \underline{E}_{0}^{r}, \underline{E}^{r} \cdot \underline{E}_{0}^{d}$ and $\underline{E}^{r} \cdot \underline{E}_{0}^{r}$ all evaluated separately. The phase structure of these components can exhibit significant differences and quite different coefficients are often needed for the analytic completion of these integrals. For regions of propagation where reflection and diffraction processes dominate, the analytic completion is often sufficiently accurate to only require a negligible region of numerical integration. Where ducting effects occur, however, the analytic completion should start a significant distance above the duct.

\section{REFRACTIVE EFFECTS}

The major aim of the current report is to further develop the above propagation procedure so that it can incorporate refractive effects in addition to those of reflection and diffraction. Problems involving refraction are normally analyzed through numerical solutions of the PE approximation to Maxwell's equations [1]. There is no reason, however, why (3) (or (1) and (2)) could not be applied to such problems. Indeed, providing that the phase distance $\phi$ and the spreading distance $s$ take account of variations in refractive index, we can still use the electric fields that result from expressions (4) and (5). The exact values for $\phi$ and $s$ will, however, require the solution of complex ray tracing equations. Phase distance $\phi$ is calculated from $\phi=\int n d s$ with integration along the ray path between the source and field observation point. In addition, the calculation of $s$ requires the deviation in rays as they move out from the source. For weak variations in refractive index, however, the ray path over a limited distance will be approximately linear and $s$ will be the geometric distance to a first approximation. Likewise, the phase distance $\phi$ will be the geometric distance to a first approximation. If $\beta$ is large, however, phase variations can be quite large even when phase distance variations are small. Consequently, at microwave 


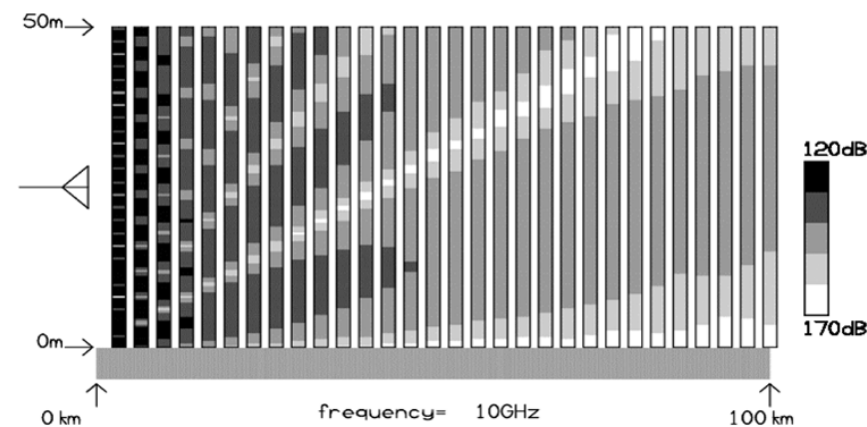

Fig. 3. Transmission losses experienced over a flat Earth sea.

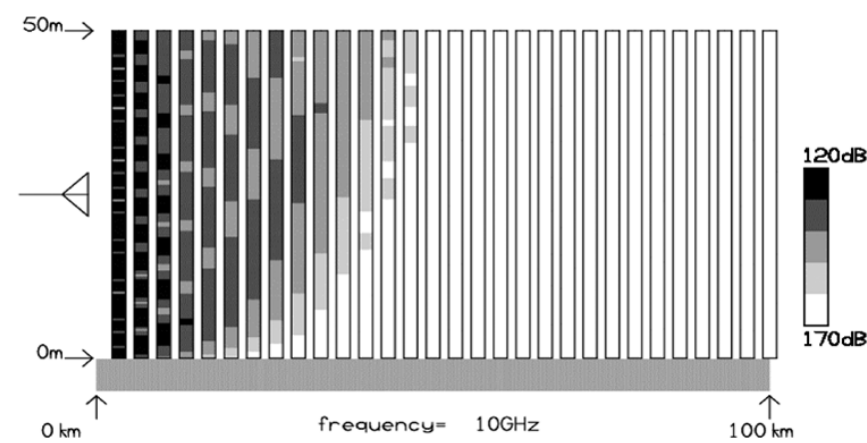

Fig. 4. Transmission losses experienced over the sea with Earth's curvature include.

frequencies, it is the phase variations of the fields that will dominate when refractivity variations are weak and we will need to calculate phase distance to higher than leading order. Fortunately, we include a higher order approximation to phase when we simply evaluate the phase distance integral along the straight line that joins the source to the field observation point. This follows from Fermat's principle which implies that a small error in the ray path will only cause a second order error in the phase distance. Consequently, for weak variation in refractive index (as is the case for the atmosphere), there is no need to solve the ray trace equations that describe the full GO solution. We simply use the geometric distance for $\mathrm{s}$ and evaluated $\phi$ along a straight line path. This results in considerable computational saving.

As an example of the effectiveness of the method, consider propagation over sea water $\left(\sigma=5 \mathrm{~S} / \mathrm{m}\right.$ and $\left.\varepsilon_{r}=81\right)$. Fig. 3 shows a simulation of transmission losses over a flat Earth for an isotropic radiator at $25 \mathrm{~m}$ above the sea level on a frequency of $10 \mathrm{GHz}$. Equivalent propagation over a curved Earth is shown in Fig. 4. The effect of Earth's curvature is simulated by means of a modified refractive index (denoted by $m$ ) in place of the unmodified refractive index (denoted by $n$ ), a convenient form of $m$ being [1] $m=1+10^{-6} \times(330+118 y)$ where $y$ is the height above sea level in meters. Fig. 4 shows good agreement with the corresponding simulation in [1]. Now consider an evaporative surface duct with modified refractive index given by [1]

$$
m=1+10^{-6} \times\left(330+\frac{1}{8}\left(y-d \ln \left(\frac{y}{y_{0}}\right)\right)\right)
$$

where $d$ is the duct height in meters and $y_{0}$ is a measure of the surface roughness (taken to be $15 \times 10^{-5} \mathrm{~m}$ in the current work). Fig. 5(a) shows some simulated propagation loss through

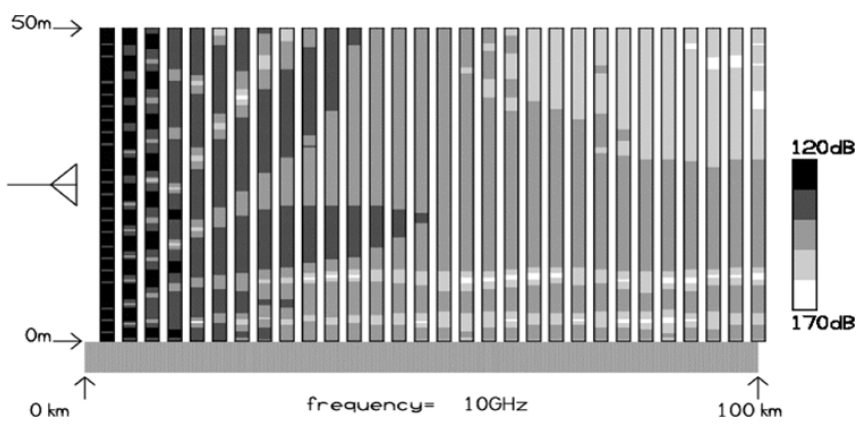

(a)

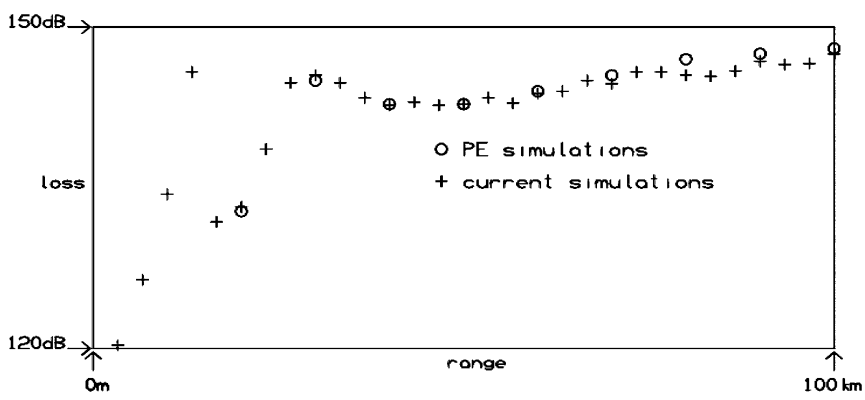

(b)

Fig. 5. (a) Transmission losses with surface duct added. (b) Boresight losses compared with accurate PE simulations from [1].

a duct of height $30 \mathrm{~m}$. It will be noted that there is significant propagation in the duct, with loss behavior that is consistent with that calculated using the PDE approach [1]. Once again there is good agreement with the corresponding simulations in [1]. More detailed comparisons are shown in Fig. 5(b) for the case of boresight and it will be noted that the PE results, extracted from [1], show good agreement with the current simulations.

It will be noted that the choice of intermediate surface spacing in the above simulations $(3.3 \mathrm{~km})$ has given results that compare well with the accurate PDE simulations of [1]. The question arises, however, as to how we choose the correct spacing in general. Basically, we need to ensure that the GO solution remains valid over the entire region between two adjacent intermediate surfaces. If $D$ represents a typical length scale (duct or building height for example) we must ensure that $\lambda \ll D$ and $\lambda L \ll D^{2}$ ( $L$ is the distance between the surfaces and $\lambda$ is the free space wavelength) for the GO solution to remain valid over the distance $L$ (see [14] for further consideration of GO validity). In practice, it was found that $L$ should be less than $D^{2} / \pi \lambda$. Consequently, for the above ducting problem, a spacing of about 9 $\mathrm{km}$ is adequate. When the approximate form of the GO solution is used, it is also necessary to check that there is no significant ray focussing over the distance $L$. In the case of atmospheric propagation, however, focusing is usually insignificant over this distance.

Fig. 6(a) shows some simulated propagation through an elevated duct. In this case, the modified refractive index takes the form

$$
\begin{aligned}
& (m-1) \times 10^{6} \\
& =330.0+0.118 \times y \quad y \leq 100 \\
& \quad=341.8-0.2 \times(y-100) \quad 100<y \leq 150 \\
& \quad=331.8+0.118 \times(y-150) \quad 150<y .
\end{aligned}
$$




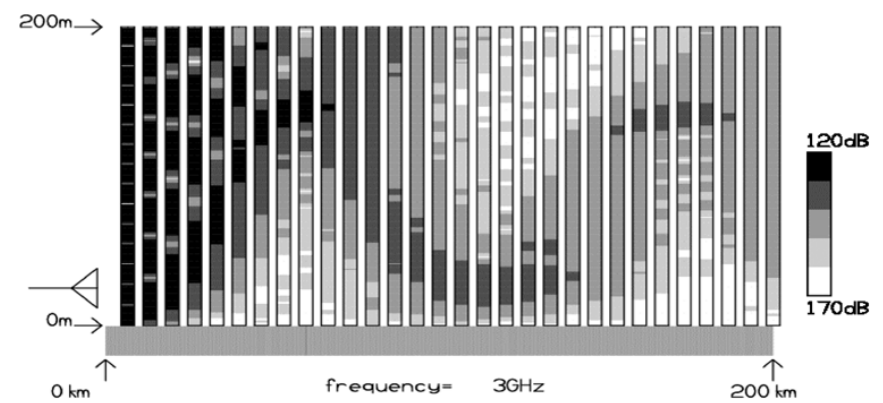

(a)

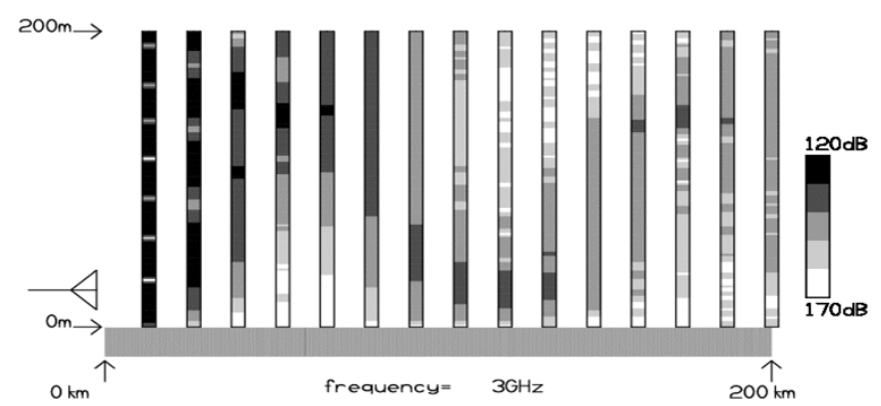

(b)

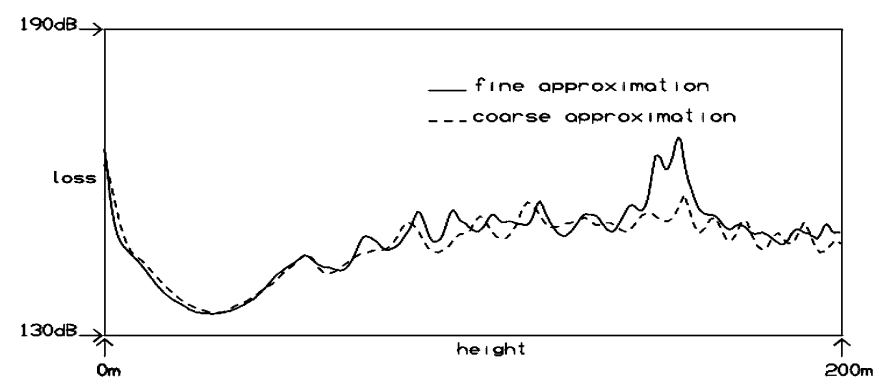

(c)

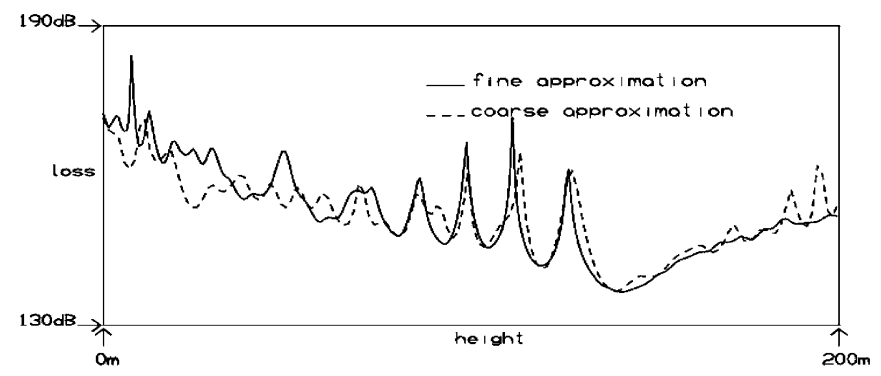

(d)

Fig. 6. (a) Transmission losses experienced in an elevated duct. (b) Transmission losses with coarse simulation. (c) Elevated duct losses at a range of $106.7 \mathrm{~km}$. (d) Elevated duct losses at a range of $166.7 \mathrm{~km}$.

It will be noted that some propagation gets trapped in a duct below the height of $150 \mathrm{~m}$, a result that is in accord with the predictions of the parabolic equation method [1]. Fig. 6(b) shows the simulated results for same problem, but with half the number of intermediate surfaces and half the number of vertical quadrature intervals. It will be noted that the results are in accord with those from the more refined simulation and illustrates the ability to take very large horizontal propagation steps. The second simulation uses a very coarse discretization but captures the main detail of the more refined simulation. This is further illustrated in Fig. 6(c) and (d) which show more detailed comparison at the ranges of 106.7 and $166.7 \mathrm{~km}$. These figures show the effect

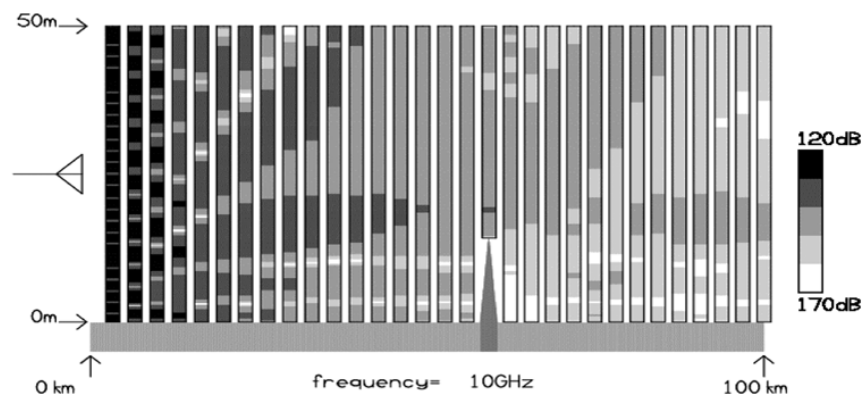

(a)

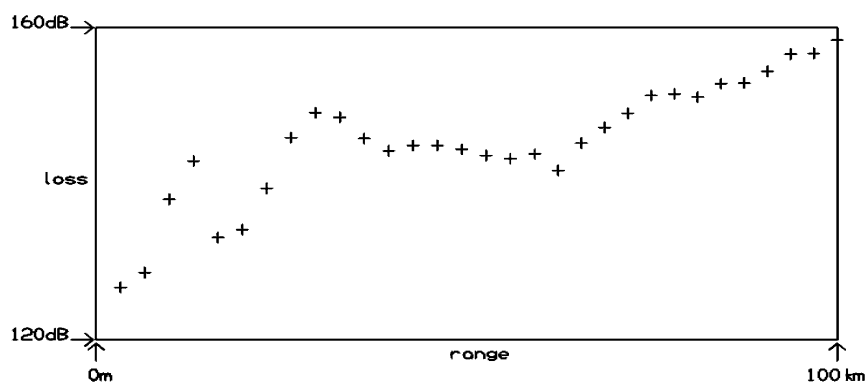

(b)

Fig. 7. (a) Surface duct that has been intercepted by an island. (b) Detailed results at the height of the island.

of the duct at two representative ranges and demonstrate good convergence for the method.

Fig. 7(a) shows a further example that includes the effects of ground topography. In this example, the surface duct of Fig. 5(a) is intercepted by a small island. It will be noted that the island causes most of the power to be scattered upwards, but that there is also some weak excitement of the duct to the lee of the island. Fig. 7(b) shows detailed simulation results at the height of the island).

\section{FURTHER REFINEMENTS}

One of the main problems with the above approach is the large computational requirement in the evaluation of integrals over the intermediate surfaces. PE methods can greatly reduce this requirement by means of split step FFT techniques, but require a far greater number of intermediate surfaces. The current approach requires far fewer intermediate surfaces, but a greater complexity in the calculations to be performed on these surfaces. The Fresnel nature of the surface integrals means that quadrature points can be fairly sparse at lower altitudes, but must increase in density with height. Eventually, the quadrature points will become too dense and the integral must be completed in an analytic manner (as described in Section III). We can extend the idea of analytic evaluation by noting that both the phase distance and amplitude of the integrand are usually slowly varying and so an approximation of the form of (6) can be effective over large intervals, even at high altitudes. Consequently, an analytic integral of such an expansion can provide an economical quadrature at these higher altitudes. Defining $M$ by

$$
\begin{aligned}
& M(a, b, c, d, e, f)=d M_{0}(a, b, c)+e M_{1}(a, b, c) \\
&+ f M_{2}(a, b, c)
\end{aligned}
$$


an analytic integral is obtained from the result

$$
\begin{aligned}
& N(a, b, c, d, e, f)= \int_{h_{1}}^{h_{2}}\left(d+e Y+f Y^{2}\right) \\
& \times \exp \left(-j\left(a Y^{2}+2 b Y+c\right)\right) d Y \\
&= M\left(a, a h_{1}+b, a h_{1}^{2}+2 b h_{1}+c,\right. \\
&\left.d+e h_{1}+f h_{1}^{2}, e+2 f h_{1}, f\right) \\
& \\
&-M\left(a, a h_{2}+b, a h_{2}^{2}+2 b h_{2}+c,\right. \\
&\left.d+e h_{2}+f h_{2}^{2}, e+2 f h_{2}, f\right) .
\end{aligned}
$$

In the current work, this approach is invoked at altitudes where the phase variations have become too large for the low altitude quadrature rule (Simpson rule in the current work) to be effective for the chosen quadrature interval. (Note that the integrand expansion coefficients are found by fitting quadratics to the phase and amplitude at quadrature points.) Such an approach allows the use of quite large quadrature intervals at all altitudes and considerably accelerates the method. Furthermore, the major contribution to the surface integrals will come from around the altitude of the observation point $\underline{r}_{0}$ in (3) and contributions at altitudes differing by more than a few $\mathrm{D}$ (the typical scale) can usually be ignored. Further savings can be achieved by calculating the field from a GO estimate at altitudes for which the field source is clearly within view. For a simple fixed quadrature on the intermediate surfaces, the complexity of the current technique will be about the same as that for a standard finite difference approximation of the PE approach. It does not, however, have the savings that PE methods derive from fast Fourier transform (FFT) techniques. The major advantage of the current approach is in the flexibility of the approximations that can be applied on the intermediate surfaces. On many of these surfaces, a few quadrature intervals will suffice and this can more than compensates for the lack of FFT techniques. Furthermore, the use of integral propagators makes it easy to blend different solution techniques. In particular, the current techniques could be used to further develop a solution that has been derived up to a given intermediate surface by another technique. In addition, for specialised parts of the propagation, it might be possible to obtain dipole solutions with far greater range of validity than the GO solutions and hence further increase the steplengths that can be achieved. A further consideration with any computer technique is its memory requirement. Being explicit, however, the requirement of the current technique is very small.

\section{CONCLUSION}

The present paper has considered an efficient approach to the estimation of radio wave propagation through environments with non homogeneous atmospheres. This approach is based on Kirchhoff style integral relations and provides an alternative to the more common split step PE methods. Unlike the PE methods, however, the current approach does not require a rectangular grid and hence has few problems with complicated boundaries. Furthermore, the approach does not require absorbing boundaries or involved patching to upper atmosphere solutions. The effectiveness of the approach has been demonstrated through its application to several non trivial problems and the results found to be in agreement with simulations performed by other approaches. Although the current paper has concentrated on a pseudo two dimensional form of the technique, it is readily extended to three dimensions and efficient methods for achieving this are under development.

\section{ACKNOWLEDGMENT}

The author would like to thank the referees for helpful comments.

\section{REFERENCES}

[1] M. Levy, Parabolic Equation Methods for Electromagnetic Wave Propagation, ser. IEE Electromagnetic wave series 45, 2000.

[2] J. R. Kuttler and G. D. Dockery, "Theoretical description of the parabolic approximation/Fourier split-step method of representing electromagnetic propagation in the troposphere," Radio Sci., vol. 26, pp. 381-393, 1991.

[3] A. E. Barrios, "A terrain parabolic equation model for propagation in the troposphere," IEEE Trans. Antennas Propag., vol. 45, no. 1, pp. 90-98, Jan. 1996.

[4] J. L. Young and R. O. Nelson, "A summary and systematic analysis of FDTD algorithms for linearly dispersive media," IEEE Antennas Propag. Mag., vol. 43, pp. 61-77, 2001.

[5] L. J. Nickisch and P. M. Franke, "Finite-Difference time domain implementation for radio wave propagation in a plasma," Radio Sci., vol. 29 , pp. 1513-1522, 1992.

[6] F. Akleman and L. Sevgi, "Realistic surface modeling for a finite-difference time-domain wave propagator," IEEE Trans. Antennas Propag., vol. 51, no. 8, pp. 1675-1679, Aug. 2003.

[7] M. O. Ozyalcin, F. Akleman, and L. Sevgi, "A novel TLM-based timedomain wave propagator," IEEE Trans. Antennas Propag., vol. 51, no. 8, pp. 1680-1682, Aug. 2003.

[8] L. Barclay, Ed., Propagation of Radio Waves, 2nd ed: IEE, 2003.

[9] J. H. Whitteker, IEEE J.Sel. Area. Comm., vol. 20, pp. 515-522, 2002.

[10] G. D. Monteath, Application of Electromagnetic Reciprocity Principle. New York: Pergamon, 1973.

[11] C. J. Coleman, "Application of the reciprocity theorem to complex propagation problems," in Proc. IEEE Antennas and Propagation Conf., Boston, MA, Jun. 2001, pp. 444-447.

[12] - "Huygens principle applied to radio wave propagation," Radio Sci., vol. 37, 2002.

[13] M. Abramowitz and I. A. Stegun, A Handbook of Mathematical Functions Dover, 1965.

[14] L. B. Felsen and N. Marcuvitz, Radiation and Scattering of Waves, ser. IEEE Press Series on Electromagnetic Propagation, 1994.

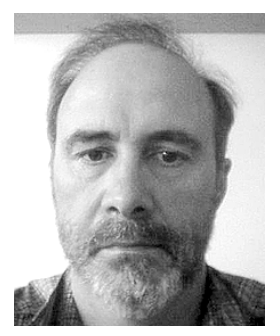

Chris J. Coleman (M'00) received the B.Sc. degree in mathematics and the Ph.D. degree in theoretical physics from Imperial College, London University, London, U.K., in 1971 and 1974, respectively, and the B.Eng. degree in electrical and electronic engineering from Adelaide University, Adelaide, South Australia, in 2000.

Between 1974 and 1990, he held a variety of teaching and research posts in the Mathematics departments of Imperial College, the University of Wales, the University of Nottingham, and the University of Wollongong. From 1990 until 1999, he served as a Principal Research Scientist on the Jindalee over the horizon radar project. In 1999, he joined the Electrical and Electronic Engineering Department of the University of Adelaide as an Associate Professor. His current research interests include radio wave propagation, antennas and mathematical methods for engineering.

Dr. Coleman is an Associate Editor for the IEEE ANTENNAS AND WIRELESS PROPAGATION LETTERS. 